$\begin{array}{ccc}\text { Available online at www.sinjas.journals.ekb.eg } & \overline{\text { ARUEGYPT }} \\ \text { SCREENED BY SINAI Journal of Applied Sciences }\end{array}$

\title{
RESPONSE OF SOME SUNFLOWER GENOTYPES TO NITROGEN FERTILIZER LEVELS
}

\author{
Saad K.M. Hafez ${ }^{1}$; M.H. Mubarak ${ }^{* 2}$; T.H.A. Hassan ${ }^{1}$ and M.N. ElBasiouny ${ }^{2}$ \\ 1. Arish Agric. Res. Station, Oil Crops Res. Dept., Field Crop Res. Inst., ARC, Egypt \\ 2. Dept. Plant Prod., Fac. Environ. Agric. Sci., Arish Univ., Egypt
}

\begin{tabular}{l} 
ARTICLE INFO \\
\hline Article history: \\
Received: 05/10/2020 \\
Revised: $20 / 02 / 2021$ \\
Accepted: $13 / 03 / 2021$ \\
Available online: 04/04/2021 \\
\hline Keywords: \\
Sunflower, \\
genotype, \\
nitrogen fertilizer.
\end{tabular}

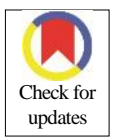

\begin{abstract}
A field study was carried out during summer seasons of 2018 and 2019 at the Experimental Farm of Faculty of Environmental Agricultural Sciences, Arish University, El-Arish, North Sinai Governorate. Egypt, This study aimed to investigate the response of three sunflower genotypes (Helianthus annus L., i.e. Giza-102, Sakha-53, Line 120 to three nitrogen levels N1=15, N2= 30, $\mathrm{N} 3=45 \mathrm{~kg} \mathrm{~N}_{\text {fed }}{ }^{-1}$ to maximize seed and oil yields of sunflower crop under the newly reclaimed soil and the environmental conditions of North Sinai. Salinity of irrigation water ranged from 4500 to $5500 \mathrm{ppm}$, using drip irrigation system. The main results were that Giza-102 genotype surpassed the other studied genotypes in plant height $(\mathrm{cm})$, stem diameter $(\mathrm{cm})$, number of leaves per plant, leaves fresh and dry weights per plant $(\mathrm{g})$, yield attributes, seed and oil yields. There were significant differences between $\mathrm{N}$-fertilizer levels found at most of growth characters, seed and oil yields per feddan. While, oil content decreased.
\end{abstract}

\section{INTRODUCTION}

Sunflower (Helianthus annuus, L.) plays an important role in solving the gap between demand and consumption of edible oil in Egypt, where, seeds contain about 40 $-45 \%$ oil, it can grow under wide environmental conditions and its roughage could be used in animal feeding. Sunflower ranked the fourth position after groundnut, soybean and rapeseed (Khandekar et al., 2018). It belongs to Asteraceae (compositeae) family and originated from the South western United States and Northern Canada (Mohamed et al., 2018).Nitrogen ( $\mathrm{N}$ ) is the most significant nutrient for improving seed and oil yields, as it is the most essential mineral nutrient and the widest application in modern agriculture (Mulvaney et al., 2009). Application $\mathrm{N}$ fertilizer levels at 45 $\mathrm{kg} \mathrm{N}$ fad. $^{-1}$ (Hassan, 2010) in Egypt; $120 \mathrm{~kg}$ $\mathrm{N} \mathrm{ha}^{-1}$ (Oyinlola et al., 2010) in Nigerian; $125 \mathrm{~kg} \mathrm{~N} \mathrm{ha}^{-1}$ in Pakistan, resulted taller plants and thicker stems as compared to the lower and/or upper $\mathrm{N}$ used levels. The increase in seed yield and its components of sunflower as the amount of nitrogen fertilizer increased may refer to enhancing vegetative growth of aerial parts and physical maturity. Maximum oil content $(41.9 \%)$ was resulted from without application of $\mathrm{N}$ fertilizer and minimum oil content (38.4\%) was observed when $240 \mathrm{~kg}$ $\mathrm{N} \mathrm{ha}{ }^{-1}$ was applied (Nasim et al., 2012). Also, El-Sarag (2007) found that $\mathrm{N}$ application improved growth and growth analyses of sunflower cultivars

\section{MATERIALS AND METHODS}

A field study was carried out during summer seasons of 2018 and 2019 at the Experimental Farm, Faculty of Environmental Agricultural Sciences, Arish University, ElArish, North Sinai Governorate, Egypt. This study aimed to investigate the response

\footnotetext{
* Corresponding author: E-mail address: mobark_mohamed99@yahoo.com https://doi.org/ 10.21608/sinjas.2021.45301.1005

(C) 2020 SINAI Journal of Applied Sciences. Published by Fac. Environ. Agric. Sci., Arish Univ. All rights reserved.
} 
of three sunflower genotypes (Helianthus annus L., i.e. Giza-102, Sakha-53, Line 120 to three nitrogen levels $(\mathrm{N} 1=15, \mathrm{~N} 2=30$, $\mathrm{N} 3=45 \mathrm{~kg} \mathrm{~N} \mathrm{fad}^{-1}$ ). Sunflower seeds were obtained from Oil Crops Research Section, Field Crops Research Institute, Agriculture Research Center, Giza, Egypt. Factorial experiment split plot design with four replications was used and included 9 treatments which were the combination of three cultivars in the main plots and the three nitrogen fertilizer levels in the subplots. Ammonium nitrate fertilizer $(33.5 \%$ $\mathrm{N})$ was the source of nitrogen in both seasons. The fertilizer quantities were divided into 8 equal portions, the first one was added after 2 weeks from sowing then the other portions were added gradually every week. The recommended rate of calcium superphosphate $\left(15.5 \% \quad \mathrm{~kg} \quad \mathrm{P}_{2} \mathrm{O}_{5}\right)$ was applied during soil preparation at the rate of $200 \mathrm{~kg}$ fad. $^{-1}$ Potassium sulfates $\left(48 \% \mathrm{~kg} \mathrm{~K}_{2} \mathrm{O}\right)$ at rate of $50 \mathrm{~kg} \mathrm{fed}^{-1}$ was applied at five equal doses, the first dose after thinning and the other doses every one week. All other agricultural practices were carried out as recommended for sunflower growing under the conditions of North Sinai. Drip irrigation system was used with water salinity of $4500-5500 \mathrm{ppm}$. The irrigation lines length was $30 \mathrm{~m}$ and between lines $50 \mathrm{~cm}$ was left to gain plot area of $10.5 \mathrm{~m}^{2}(3.5 \mathrm{~m}$ long $\times 3 \mathrm{~m}$ wide, which gave approximately 84 plants/ plot with $25 \mathrm{~cm}$ between drippers).The planting date was on $23^{\text {th }}$ May in 2018 and 2019 seasons. The harvesting dates for the three genotypes Giza-102, Sakha-53, Line- 120 were after 73,83 and 85 days, respectively. Soil texture was sandy and total $\mathrm{N}$ were 10 and $13 \mathrm{ppm}$ with $\mathrm{pH}$ average 7.6 according to the soil mechanical analysis in both seasons. Samples of five guarded plants from each experimental plot were collected randomly at 30,40, 50 and 60 days after sowing for studying the effects of the applied treatments on vegetative characters (plant height, stem diameter, number of leaves/plant, fresh and dry weights for leaves, No. head per plant).At the end of heading, the heads of the three inner rows were bagged at early seed development for avoiding bird damages and were used for estimating yield and its components as well as seed oil content. Ten guarded plants were taken randomly from each experimental plot for measuring head diameter $(\mathrm{cm}), 100$ - seed weight $(\mathrm{g})$, seed weight per plant $(\mathrm{g})$, seed yield per $\mathrm{m}^{-2}$ then seed yield (ton fad. $^{-1}$ ) was calculated. Seed oil content (\%) was determined by using Soxhlet method with 6-syphones according to the AOAC (1990). Data were statistically analyzed according to Snedecor and Cochran (1956) using MSTAT computer program V.4 (1986). The means values were compared at 0.05 level of probability using Duncans Multiple Range Test (Duncan, 1955).

\section{RESULTS AND DISCUSSION}

Results in Tables 1, 2, 3, 4, 5 and 6 show the response of sunflower genotypes (Sakha-53, Line 120, Giza-102) to nitrogen fertilizer levels $\left(15,30\right.$ and $45 \mathrm{~kg} \mathrm{~N}$ fad. $\left.{ }^{-1}\right)$ and their interactions at different growth periods (after 30, 40, 50 and 60 days after sowing DAS and seed yield, yield components and seed oil contents in two summer seasons $(2018,2019)$.

\section{Plant Height (cm)}

\section{Genotypes variation}

There are significant differences among sunflower genotypes regarding plant height, stem diameter, number of leaves per plant, leaves fresh weight $(\mathrm{g})$ and Leaves dry weight $(\mathrm{g})$ at most of samples in both seasons (Table 1). Giza-102 gave the highest value of plant height $(61.85,83.91$, 129.00 and $174.50 \mathrm{~cm}$ ) in the first seasons, the same trend was recorded in the second season where in the highest value of plant height recoded 80.50, 90.13, 103.20 and $179.70 \mathrm{~cm}$ at $30,40,50$ and 60 days, respectively. 
Table 1. Effect of genotypes and nitrogen levels on plant height $(\mathrm{cm})$ of sunflower at 30, 40, 50 and 60 days after sowing in 2018 and 2019 seasons

\begin{tabular}{|c|c|c|c|c|c|c|c|c|c|}
\hline Vegetative & tment & & ant heigh & t $(\mathrm{cm}) 201$ & & & Plant heig & ht (cm) 20 & \\
\hline Days & & 30 & 40 & 50 & 60 & 30 & 40 & 50 & 60 \\
\hline Genotype & Sakha-53 & $52.17 \mathrm{ab}$ & 79.21ab & $113.90 \mathrm{~b}$ & $170.10 b$ & $71.00 \mathrm{c}$ & $82.74 b$ & $93.87 \mathrm{c}$ & $167.90 \mathrm{c}$ \\
\hline & Line-120 & $49.95 \mathrm{c}$ & $78.57 b$ & $117.80 \mathrm{ab}$ & $171.20 \mathrm{ab}$ & $75.57 b$ & $85.06 \mathrm{ab}$ & $96.43 b$ & $172.50 \mathrm{~b}$ \\
\hline & Giza-102 & $61.85 \mathrm{a}$ & $83.91 \mathrm{a}$ & $129.00 \mathrm{a}$ & $174.50 \mathrm{a}$ & $80.50 \mathrm{a}$ & $90.13 a$ & $103.20 \mathrm{a}$ & $179.70 \mathrm{a}$ \\
\hline Nitrogen level & $15 \mathrm{~N}$ & $57.42 \mathrm{~b}$ & $78.31 b$ & $98.90 \mathrm{~b}$ & $116.90 \mathrm{~b}$ & $63.48 c$ & $83.46 \mathrm{~b}$ & $93.87 \mathrm{c}$ & $166.30 \mathrm{~b}$ \\
\hline & $30 \mathrm{~N}$ & $58.33 \mathrm{ab}$ & $80.81 b$ & $102.50 \mathrm{ab}$ & $136.00 \mathrm{ab}$ & $71.94 \mathrm{~b}$ & $84.50 \mathrm{ab}$ & $96.43 \mathrm{~b}$ & $173.20 \mathrm{ab}$ \\
\hline & $45 \mathrm{~N}$ & $59.83 \mathrm{a}$ & $84.57 \mathrm{a}$ & $119.20 \mathrm{a}$ & $174.80 \mathrm{a}$ & $77.65 \mathrm{a}$ & $86.96 \mathrm{a}$ & $103.20 \mathrm{a}$ & $178.60 \mathrm{a}$ \\
\hline
\end{tabular}

Means having the same letter within each column are not significantly differed at 0.05 level, according to Duncan's multiple range test.

\section{Effect of nitrogen levels}

There are significant effects of nitrogen levels on plant height at all growth stages studied of sunflower in both seasons (Table 1). The tallest plants $(59.83,84.57,119.20$ and $174.80 \mathrm{~cm}$ ) in the first seasons, and $(77.65,86.96,103.20$ and $178.60 \mathrm{~cm})$ in the $2^{\text {nd }}$ season at $30,40,50$ and 60 days, respectively, were obtained under nitrogen level of $45 \mathrm{~kg}$ fad.

\section{Effect of interaction}

Results in Table 2 show significant effect of genotypes $\times$ nitrogen levels interaction on plant height at 30, 40, 50 and 60 days in 2018 and 2019 seasons where in the tallest plants recorded 77.17, 96.50, 141.70 and $183.00 \mathrm{~cm}$ in the first seasons; and $88.00,106.33,122.30$ and 184.60 in the $2^{\text {nd }}$ season. The same results were by with Awad and Gharib (2009), Abd ElMotagally and Osman (2010), Oyinloa et al. (2010), Irika (2015), Ravishankar and Malligawad (2017) and Khandekar et al. (2018). They placed that application of various nitrogen mineral fertilizer levels was followed by a significant increase in plant height $(\mathrm{cm})$.

\section{Stem Diameter $(\mathrm{cm})$}

\section{Genotypes variation}

There are significant differences among sunflower genotypes in stem diameter $(\mathrm{cm})$ at most of samples in both seasons (Table 3). Sunflower cultivar Giza-102 gave the highest value for stem diameter (1.356, $1.551,1.732$ and $1.891 \mathrm{~cm}$ ) in the first seasons, the same trend was recorded in the second season and the values were 1.500, $1.600,1.774$ and $1.956 \mathrm{~cm}$ at $30,40,50$ and 60 days, respectively.

\section{Effect of nitrogen levels}

There are significant effects of nitrogen levels on stem diameter at all growth stages studied of sunflower in both seasons (Table $3)$. The heights values of stem diameter in the first seasons $(1.344,1.537,1.659$ and $1.833 \mathrm{~cm})$ in the second seasons and $(1.467$, $1.556,1.811$ and $1.930 \mathrm{~cm}$ ) in the second seasons at 30, 40, 50 and 60 DAS, respectively were the resultant of nitrogen treatment $45 \mathrm{~kg} / \mathrm{fad}$.

\section{Effect of interaction}

Results in Table 4 show significant effect of genotypes $\times$ nitrogen levels interaction on 
Table 2. Effect of interaction among genotypes and nitrogen levels on plant height $(\mathrm{cm})$ of sunflower at 30, 40, 50 and 60 days after sowing in 2018 and 2019 seasons

\begin{tabular}{|c|c|c|c|c|c|c|c|c|c|}
\hline \multirow{2}{*}{\multicolumn{2}{|c|}{$\begin{array}{c}\text { Genotype Nitrogen levels } \\
\text { Days }\end{array}$}} & \multicolumn{4}{|c|}{ Plant height (cm) 2018} & \multicolumn{4}{|c|}{ Plant height (cm) 2019} \\
\hline & & 30 & 40 & 50 & 60 & 30 & 40 & 50 & 60 \\
\hline \multirow{4}{*}{ Sakha-53 } & $15 \mathrm{~N}$ & 39.33hi & $70.50 \mathrm{~cd}$ & $106.00 \mathrm{k}-\mathrm{o}$ & $162.50 \mathrm{kl}$ & $67.001 \mathrm{mn}$ & $72.17 \mathrm{kl}$ & $85.33 \mathrm{jkl}$ & $161.40 \mathrm{jkl}$ \\
\hline & $30 \mathrm{~N}$ & $48.33 \mathrm{e}-\mathrm{i}$ & $72.50 \mathrm{bcd}$ & 95.67 no & $170.50 \mathrm{e}-\mathrm{i}$ & $71.67 \mathrm{~h}-1$ & $79.00 \mathrm{~g}-\mathrm{j}$ & $95.50 \mathrm{f}-\mathrm{i}$ & 171.50fgh \\
\hline & $45 \mathrm{~N}$ & $53.33 \mathrm{~d}-\mathrm{h}$ & h 77.33a-d & $116.30 \mathrm{f}-1$ & $173.20 \mathrm{def}$ & $75.67 d-j$ & $81.83 \mathrm{e}-\mathrm{h}$ & $98.83 \mathrm{e}-\mathrm{i}$ & 174.00def \\
\hline & $15 \mathrm{~N}$ & $47.50 \mathrm{f}-\mathrm{i}$ & $80.00 \mathrm{a}-\mathrm{d}$ & $112.20 \mathrm{j}-\mathrm{m}$ & $174.30 \mathrm{cde}$ & $76.50 \mathrm{~d}-\mathrm{i}$ & $82.33 \mathrm{e}-\mathrm{h}$ & $96.33 \mathrm{f}-\mathrm{i}$ & 171.20fgh \\
\hline \multirow[t]{3}{*}{ Line-120 } & $30 \mathrm{~N}$ & $57.67 \mathrm{~b}-\mathrm{g}$ & g 84.17a-d & $116.00 \mathrm{~g}-1$ & $175.50 \mathrm{~b}-\mathrm{e}$ & $77.00 \mathrm{c}-\mathrm{i}$ & $85.00 \mathrm{~d}-\mathrm{h}$ & $104.80 c-f$ & $177.80 \mathrm{bcd}$ \\
\hline & $45 \mathrm{~N}$ & $69.00 \mathrm{abc}$ & c88.83abc & $133.20 \mathrm{a}-\mathrm{e}$ & $178.50 \mathrm{abc}$ & $83.00 \mathrm{abc}$ & $88.67 \mathrm{cde}$ & $109.70 \mathrm{bcd}$ & $179.50 \mathrm{bc}$ \\
\hline & $15 \mathrm{~N}$ & $60.83 \mathrm{~b}-\mathrm{g}$ & g 88.83abc & 129.70b-h & $175.50 \mathrm{~b}-\mathrm{e}$ & $78.67 \mathrm{c}-\mathrm{g}$ & $85.00 \mathrm{~d}-\mathrm{h}$ & $102.00 \mathrm{~d}-\mathrm{h}$ & 171.10fgh \\
\hline \multirow[t]{2}{*}{ Giza-102 } & $30 \mathrm{~N}$ & $62.33 b-e$ & e $94.00 \mathrm{ab}$ & $134.00 \mathrm{a}-\mathrm{d}$ & $180.30 \mathrm{ab}$ & 81.33b-e & $88.33 \mathrm{cde}$ & $114.20 \mathrm{ab}$ & $180.80 \mathrm{~b}$ \\
\hline & $45 \mathrm{~N}$ & $77.17 \mathrm{a}$ & $96.50 \mathrm{a}$ & $141.70 \mathrm{a}$ & $183.00 \mathrm{a}$ & $88.00 \mathrm{a}$ & $106.33 \mathrm{a}$ & $122.30 \mathrm{a}$ & $184.40 \mathrm{a}$ \\
\hline
\end{tabular}

Means having the same letter within each column are not significantly differed at 0.05 level, according to Duncan's multiple range test.

Table 3. Effect of genotypes and nitrogen levels on stem diameter $(\mathrm{cm})$ of sunflower at 30, 40, 50 and 60 days after sowing in 2018 and 2019 seasons

\begin{tabular}{|c|c|c|c|c|c|c|c|c|c|}
\hline \multirow{2}{*}{$\begin{array}{l}\text { Vegetative } \\
\text { characters } \\
\text { Days }\end{array}$} & \multirow[t]{2}{*}{ Treatment } & \multicolumn{4}{|c|}{ stem diameter $(\mathrm{cm}) 2018$} & \multicolumn{4}{|c|}{ stem diameter $(\mathrm{cm}) 2019$} \\
\hline & & 30 & 40 & 50 & 60 & 30 & 40 & 50 & 60 \\
\hline \multirow[t]{3}{*}{ Genotype } & Sakha-53 & $1.211 \mathrm{c}$ & $1.48 \mathrm{c}$ & $1.62 \mathrm{c}$ & $1.76 \mathrm{c}$ & $1.330 \mathrm{c}$ & $1.459 \mathrm{c}$ & $1.644 \mathrm{c}$ & $1.844 \mathrm{c}$ \\
\hline & Line- 120 & $1.296 b$ & $1.49 \mathrm{~b}$ & $1.65 b$ & $1.82 \mathrm{ab}$ & $1.374 b$ & $1.481 \mathrm{~b}$ & $1.659 \mathrm{~b}$ & $1.890 \mathrm{~b}$ \\
\hline & Giza-102 & $1.356 \mathrm{a}$ & $1.551 \mathrm{a}$ & $1.732 \mathrm{a}$ & $1.891 \mathrm{a}$ & $1.500 \mathrm{a}$ & $1.600 \mathrm{a}$ & 1.774 & $1.956 \mathrm{a}$ \\
\hline \multirow[t]{3}{*}{ Nitrogen level } & $15 \mathrm{~N}$ & $1.265 \mathrm{c}$ & $1.504 \mathrm{c}$ & $1.610 \mathrm{c}$ & $1.737 \mathrm{c}$ & $1.341 \mathrm{c}$ & $1.433 \mathrm{c}$ & $1.722 \mathrm{c}$ & $1.874 \mathrm{c}$ \\
\hline & $30 \mathrm{~N}$ & $1.310 \mathrm{ab}$ & $1.515 \mathrm{ab}$ & $1.620 \mathrm{~b}$ & $1.800 \mathrm{~b}$ & $1.396 \mathrm{~b}$ & $1.463 b$ & $1.796 b$ & $1.885 b$ \\
\hline & $45 \mathrm{~N}$ & $1.344 \mathrm{a}$ & $1.537 \mathrm{a}$ & $1.659 \mathrm{a}$ & $1.833 \mathrm{a}$ & $1.467 \mathrm{a}$ & $1.556 \mathrm{a}$ & $1.811 \mathrm{a}$ & $1.930 \mathrm{a}$ \\
\hline
\end{tabular}

Means having the same letter within each column are not significantly differed at 0.05 level, according to Duncan's multiple range test.

Table 4. Effect of interaction between genotypes and nitrogen levels on stem diameter (cm) of sunflower at 30, 40, 50 and 60 days after sowing in 2018 and 2019 seasons

\begin{tabular}{|c|c|c|c|c|c|c|c|c|c|}
\hline \multirow[t]{2}{*}{ Genotype } & \multirow{2}{*}{$\begin{array}{l}\text { Nitrogen level } \\
\text { Days }\end{array}$} & \multicolumn{4}{|c|}{ Stem diameter(cm) 2018} & \multicolumn{4}{|c|}{ Stem diameter(cm)2019 } \\
\hline & & 30 & 40 & 50 & 60 & 30 & 40 & 50 & 60 \\
\hline \multirow{4}{*}{ Sakha-53 } & $15 \mathrm{~N}$ & $1.117 \mathrm{fg}$ & $1.47 \mathrm{cde}$ & $1.63 b-f$ & 1.70def & $1.233 \mathrm{e}-\mathrm{h}$ & $1.300 \mathrm{ij}$ & $1.567 \mathrm{hij}$ & $1.800 \mathrm{fg}$ \\
\hline & $30 \mathrm{~N}$ & $1.100 \mathrm{~g}$ & $1.40 \mathrm{ef}$ & $1.57 \mathrm{def}$ & 1.70def & $1.167 \mathrm{fgh}$ & $1.367 \mathrm{~g}-\mathrm{j}$ & $1.633 \mathrm{f}-\mathrm{i}$ & $1.867 \mathrm{~d}-\mathrm{g}$ \\
\hline & $45 \mathrm{~N}$ & $1.167 \mathrm{~d}-\mathrm{g}$ & $1.43 \mathrm{def}$ & $1.57 \mathrm{def}$ & $1.73 \mathrm{def}$ & $1.400 \mathrm{~b}-\mathrm{e}$ & $1.567 \mathrm{~b}-\mathrm{e}$ & $1.700 \mathrm{~d}-\mathrm{g}$ & $1.900 \mathrm{c}-\mathrm{f}$ \\
\hline & $15 \mathrm{~N}$ & $1.317 \mathrm{~b}-\mathrm{e}$ & $1.43 \mathrm{def}$ & $1.60 \mathrm{c}-\mathrm{f}$ & $1.70 \mathrm{def}$ & $1.333 \mathrm{c}-\mathrm{f}$ & $1.467 \mathrm{~d}-\mathrm{h}$ & $1.600 \mathrm{~g}-\mathrm{j}$ & $1.900 \mathrm{c}-\mathrm{f}$ \\
\hline \multirow[t]{3}{*}{ Line-120 } & $30 \mathrm{~N}$ & $1.450 \mathrm{abc}$ & $1.53 \mathrm{bcd}$ & $1.83 \mathrm{ab}$ & $1.90 \mathrm{bcd}$ & $1.400 \mathrm{~b}-\mathrm{e}$ & $1.500 \mathrm{~d}-\mathrm{g}$ & $1.667 \mathrm{e}-\mathrm{h}$ & $1.900 \mathrm{c}-\mathrm{f}$ \\
\hline & $45 \mathrm{~N}$ & $1.483 \mathrm{ab}$ & $1.60 \mathrm{ab}$ & $1.80 \mathrm{abc}$ & $1.90 \mathrm{bc}$ & $1.467 \mathrm{bcd}$ & $11.600 \mathrm{a}-\mathrm{d}$ & $1.667 \mathrm{e}-\mathrm{h}$ & $1.933 b-e$ \\
\hline & $15 \mathrm{~N}$ & $1.233 \mathrm{~d}-\mathrm{g}$ & $1.50 \mathrm{~b}-\mathrm{e}$ & $1.63 b-f$ & $1.90 \mathrm{bcd}$ & $1.367 \mathrm{cde}$ & $1.500 \mathrm{~d}-\mathrm{g}$ & $1.667 \mathrm{e}-\mathrm{h}$ & $1.933 \mathrm{~b}-\mathrm{e}$ \\
\hline \multirow[t]{2}{*}{ Giza-102 } & $30 \mathrm{~N}$ & $1.317 \mathrm{~b}-\mathrm{e}$ & $1.53 \mathrm{bcd}$ & $1.70 \mathrm{a}-\mathrm{f}$ & $1.90 \mathrm{bcd}$ & $1.500 \mathrm{bc}$ & $1.600 \mathrm{a}-\mathrm{d}$ & $1.800 \mathrm{a}-\mathrm{d}$ & $2.00 \mathrm{bc}$ \\
\hline & $45 \mathrm{~N}$ & $1.567 \mathrm{a}$ & $1.670 \mathrm{a}$ & $1.900 \mathrm{a}$ & $2.130 \mathrm{a}$ & $1.700 \mathrm{a}$ & $1.733 \mathrm{a}$ & $1.900 \mathrm{a}$ & $2.133 \mathrm{a}$ \\
\hline
\end{tabular}

Means having the same letter within each column are not significantly differed at 0.05 level, according to Duncan's multiple range test. 
the stem diameter at 30, 40, 50 and 60 DAs in 2018 and 2019 seasons. The largest stem diameter $(1.567,1.670,1.900$ and 2.130 $\mathrm{cm})$ in the first seasons, in the second seasons gave and $(1.700,1.733,1.900$ and $2.133 \mathrm{~cm}$ ) in the second on at 30, 40, 50 and 60 DAS after sowing, respectively, were recorded under the interaction treatment included sowing sunflower cultivar Giza-102 and application level was followed by a significant increase in stem diameter $(\mathrm{cm})$ in both seasons.

\section{Genotypes Variation}

\section{Number of leaves per plant}

There are significant differences among sunflower genotypes at most of samples in both seasons Table 5. Giza-102 gave the highest value for each of number of leaves per plant (15.04, 17.70, 19.89 and 22.98) in the first seasons, the same trend was recorded in the second season gave the highest value for each of number of leaves per plant $(16.28,19.24,21.81$ and 25.35) at $30,40,50$ and 60 days, respectively.

\section{Effect of nitrogen levels}

There are significant effects of nitrogen levels on number of leaves/plant at all growth stages studied of sunflower in both seasons (Table 5). Application of the highest $\mathrm{N}$ level (45 kg N/fad.) produced the largest number of leaves per plant (13.63, 17.50, 20.19 and 23.43) in the first seasons and 15.26, 19.37, 21.69 and 24.83 at 30, 40, 50 and 60 DAS, respectively.

\section{Effect of interaction}

Results in Table 6 show significant effect of genotypes $\mathrm{x}$ nitrogen levels interaction on number of leaves/plant at 30,40,50 and 60 DAS in 2018 and 2019 seasons. The largest number of leaves per plant 19.67 , 21.17, 21.83 and 26.17 in the first seasons, $18.50,21.17,23.17$ and 27.33 at $30,40,50$ and 60 DAS after respectively were recorded under the impact of sowing Giza102 cultivar and supplying 45kg N/fad.
The same results were found by Hassan (2010), Oyinlola et al. (2010), Hama (2015), Irika (2015) and Kandil et al. (2017). The opposite trend was observed by Abaza (2010).

\section{Leaves Fresh Weight}

\section{Genotypes variation}

There are significant differences among sunflower genotypes in their leaves fresh weight at most of samples in both seasons (Table 7). Giza-102 gave the highest leaves fresh weight $(78.55,95.34,110.83$ and $133.17 \mathrm{~g}$.) in the first seasons, the same trend was recorded in the second season, wherein the highest values of leaves fresh weight were $92.20,105.17,117.18$ and $137.47 \mathrm{~g}$ at 30, 40, 50 and $60 \mathrm{DAS}$, respectively.

\section{Effect of nitrogen levels}

There are significant effects of nitrogen levels on leaves fresh weight at all growth stages studied of sunflower in both seasons (Table 7). The heights values of leaves fresh weight $(93.87,117.30,135.14$ and 159.14) in the first seasons, as well as $109.33,135.65,147.13$ and 180.41 ) at 30 , 40, 50 and 60 DAS, respectively were the resulted of adding $45 \mathrm{~kg} \mathrm{~N} / \mathrm{fad}$.

\section{Effect of interaction}

Results in Table 8 show significant effect of genotypes $\mathrm{x}$ nitrogen levels on interaction leaves fresh weight at 30, 40, 50 and 60 DAS in 2018 and 2019 seasons. The largest leaves fresh weight (123.36, 148.32, 189.13 and 206.23g) in the first seasons, as well as $(135.47,175.27,205.11$ and $240.16 \mathrm{~g})$ in the ${ }_{2}$ nd season at 30, 40, 50 and 60 days after sowing, respectively. The same results were found by Hassan (2010). They found adding nitrogento mineral fertilizers level was followed by a significant increase in leaves fresh weight in.

\section{Leaves Dry Weight}

\section{Genotypes variation}

There are significant differences among sunflower genotypes at most of samples in both seasons (Table 9). Giza-102 sunflower 
Table 5. Effect of genotypes and nitrogen levels on number of leaves/plant of sunflower at 30, 40, 50 and 60 days after sowing in 2018 and 2019 seasons

\begin{tabular}{|c|c|c|c|c|c|c|c|c|c|}
\hline \multirow{2}{*}{$\begin{array}{l}\begin{array}{l}\text { Vegetative } \\
\text { characters }\end{array} \\
\text { Days } \\
\end{array}$} & \multirow{2}{*}{$\begin{array}{l}\text { Treatment } \\
S \\
\end{array}$} & \multicolumn{4}{|c|}{ Number of leaves/ plant 2018} & \multicolumn{4}{|c|}{ Number of leaves/ plant 2019} \\
\hline & & 30 & 40 & 50 & 60 & 30 & 40 & 50 & 60 \\
\hline \multirow[t]{3}{*}{ Genotype } & Sakha-53 & $12.72 \mathrm{c}$ & $15.59 \mathrm{c}$ & $19.17 \mathrm{bc}$ & $21.76 \mathrm{c}$ & $14.41 b$ & $18.46 b$ & $21.00 \mathrm{~b}$ & $23.17 b$ \\
\hline & Line-120 & $13.09 \mathrm{~b}$ & $16.78 b$ & $19.33 b$ & $22.43 b$ & $15.26 b$ & $18.91 b$ & $21.31 b$ & $24.00 \mathrm{ab}$ \\
\hline & Giza-102 & $15.04 \mathrm{a}$ & $17.70 \mathrm{a}$ & $19.89 \mathrm{a}$ & $22.98 \mathrm{a}$ & $16.28 \mathrm{a}$ & $19.24 \mathrm{a}$ & $21.81 \mathrm{a}$ & $25.35 \mathrm{a}$ \\
\hline \multirow[t]{3}{*}{ Nitrogen level } & $15 \mathrm{~N}$ & $13.23 b$ & $16.09 \mathrm{~b}$ & 19.20 & $21.78 b$ & $14.81 \mathrm{~b}$ & $18.30 \mathrm{~b}$ & $20.63 b$ & $22.41 b$ \\
\hline & $30 \mathrm{~N}$ & $13.35 b$ & $16.48 b$ & 19.00 & $21.96 b$ & $15.87 \mathrm{~b}$ & $18.94 b$ & $21.81 b$ & $25.28 \mathrm{ab}$ \\
\hline & $45 \mathrm{~N}$ & $13.63 \mathrm{a}$ & $17.50 \mathrm{a}$ & $20.19 b$ & $23.43 \mathrm{a}$ & $15.26 \mathrm{a}$ & $19.37 \mathrm{a}$ & $21.69 a$ & $24.83 \mathrm{a}$ \\
\hline
\end{tabular}

Means having the same letter within each column are not significantly differed at 0.05 level, according to Duncan's multiple range test.

Table 6. Effect of interaction between genotypes and nitrogen levels on number of leaves plant of sunflower at 30, 40, 50 and 60 days after sowing in 2018 and 2019 seasons

\begin{tabular}{|c|c|c|c|c|c|c|c|c|c|}
\hline \multirow{2}{*}{\multicolumn{2}{|c|}{$\frac{\text { Genotype Nitrogen level }}{\text { Days }}$}} & \multicolumn{4}{|c|}{ Number of leaves /plant 2018} & \multicolumn{4}{|c|}{ Number of leaves/ plant 2019} \\
\hline & & 30 & 40 & 50 & 60 & 30 & 40 & 50 & 60 \\
\hline \multirow{4}{*}{ Sakha-53 } & $15 \mathrm{~N}$ & $15.00 \mathrm{~cd}$ & 17.00hi & $18.83 \mathrm{e}-\mathrm{h}$ & $20.83 g-j$ & $12.83 \mathrm{kl}$ & 17.00hi & $19.50 \mathrm{kl}$ & $21.33 \mathrm{kl}$ \\
\hline & $30 \mathrm{~N}$ & $15.83 \mathrm{bcd}$ & $18.00 \mathrm{~d}-\mathrm{h}$ & $19.17 \mathrm{~d}-\mathrm{h}$ & $22.33 \mathrm{~d}-\mathrm{h}$ & $13.83 \mathrm{ijk}$ & $19.00 \mathrm{~d}-\mathrm{h}$ & $21.00 \mathrm{f}-\mathrm{j}$ & 23.00hij \\
\hline & $45 \mathrm{~N}$ & $16.67 \mathrm{a}-\mathrm{d}$ & $18.00 \mathrm{abc}$ & 19.50def & $22.50 \mathrm{~d}-\mathrm{g}$ & $14.33 \mathrm{~h}-\mathrm{k}$ & $20.00 \mathrm{a}-\mathrm{e}$ & $22.17 b-f$ & $23.67 \mathrm{c}-\mathrm{g}$ \\
\hline & $15 \mathrm{~N}$ & $17.33 \mathrm{abc}$ & $18.50 \mathrm{e}-\mathrm{h}$ & $18.83 \mathrm{e}-\mathrm{h}$ & $22.83 \mathrm{c}-\mathrm{g}$ & $14.17 \mathrm{~h}-\mathrm{k}$ & $18.50 \mathrm{e}-\mathrm{h}$ & $21.00 \mathrm{f}-\mathrm{j}$ & $23.83 \mathrm{e}-\mathrm{i}$ \\
\hline \multirow[t]{3}{*}{ Line-120 } & $30 \mathrm{~N}$ & $18.00 \mathrm{abc}$ & $19.17 \mathrm{c}-\mathrm{g}$ & $21.17 \mathrm{abc}$ & $24.67 \mathrm{abc}$ & $15.83 \mathrm{~d}-\mathrm{h}$ & $19.17 \mathrm{c}-\mathrm{g}$ & $22.00 b-f$ & $24.83 b-f$ \\
\hline & $45 \mathrm{~N}$ & $18.33 \mathrm{ab}$ & 20.50a-e & $20.98 \mathrm{a}-\mathrm{d}$ & $25.33 \mathrm{ab}$ & $16.33 \mathrm{c}-\mathrm{g}$ & 20.50a-e & $22.83 \mathrm{a}-\mathrm{d}$ & $26.17 \mathrm{abc}$ \\
\hline & $15 \mathrm{~N}$ & $17.67 \mathrm{abc}$ & $18.83 \mathrm{~d}-\mathrm{h}$ & $20.00 \mathrm{~b}-\mathrm{e}$ & $22.67 \mathrm{~d}-\mathrm{g}$ & $15.67 \mathrm{~d}-\mathrm{h}$ & $18.83 \mathrm{~d}-\mathrm{h}$ & $21.50 \mathrm{e}-\mathrm{h}$ & $24.17 b-f$ \\
\hline \multirow[t]{2}{*}{ Giza-102 } & $30 \mathrm{~N}$ & $18.00 \mathrm{abc}$ & $20.17 \mathrm{a}-\mathrm{e}$ & $20.50 \mathrm{a}-\mathrm{d}$ & 23.50 cde & $15.83 \mathrm{~d}-\mathrm{h}$ & $20.17 \mathrm{a}-\mathrm{e}$ & $22.00 b-f$ & $25.17 \mathrm{ab}$ \\
\hline & $45 \mathrm{~N}$ & $19.67 \mathrm{a}$ & $21.17 \mathrm{a}$ & $21.83 \mathrm{a}$ & $26.17 \mathrm{a}$ & $18.50 \mathrm{a}$ & $21.17 \mathrm{abc}$ & $23.17 \mathrm{a}$ & $27.33 \mathrm{a}$ \\
\hline
\end{tabular}

Means having the same letter within each column are not significantly differed at 0.05 level, according to Duncan's multiple range test.

Table 7. Effect of genotypes and nitrogen levels on leaves fresh weight (g) of sunflower at 30, 40, 50 and 60 days after sowing in 2018 and 2019 seasons

\begin{tabular}{|c|c|c|c|c|c|c|c|c|c|}
\hline $\begin{array}{l}\text { Vegetative } \\
\text { characters }\end{array}$ & \multirow[t]{2}{*}{ Treatment } & \multicolumn{4}{|c|}{ Leaves fresh weight (g) 2018} & \multicolumn{4}{|c|}{ Leaves fresh weight (g) 2019} \\
\hline$\overline{\text { Days }}$ & & 30 & 40 & 50 & 60 & 30 & 40 & 50 & 60 \\
\hline \multirow[t]{3}{*}{ Genotype } & Sakha-53 & $63.42 \mathrm{~b}$ & $73.55 \mathrm{~b}$ & $85.73 \mathrm{~b}$ & $103.17 \mathrm{~b}$ & $71.99 \mathrm{~b}$ & $85.93 \mathrm{~b}$ & $95.13 \mathrm{~b}$ & $113.30 \mathrm{~b}$ \\
\hline & Line-120 & $57.12 \mathrm{c}$ & $65.87 \mathrm{c}$ & $77.42 \mathrm{c}$ & $88.22 \mathrm{c}$ & $67.85 \mathrm{c}$ & $76.47 \mathrm{c}$ & $87.26 \mathrm{c}$ & $97.22 \mathrm{c}$ \\
\hline & Giza-102 & $78.55 \mathrm{a}$ & $95.34 \mathrm{a}$ & $110.83 \mathrm{a}$ & $133.17 \mathrm{a}$ & $92.20 \mathrm{a}$ & $105.17 \mathrm{a}$ & $117.18 \mathrm{a}$ & $137.47 \mathrm{a}$ \\
\hline \multirow[t]{3}{*}{ Nitrogen level } & $15 \mathrm{~N}$ & $45.96 \mathrm{c}$ & $55.72 \mathrm{c}$ & $67.92 \mathrm{c}$ & $77.42 \mathrm{c}$ & $57.43 \mathrm{c}$ & $65.87 \mathrm{c}$ & $75.97 \mathrm{c}$ & $87.62 \mathrm{c}$ \\
\hline & $30 \mathrm{~N}$ & $65.72 \mathrm{~b}$ & $79.96 \mathrm{~b}$ & $97.61 \mathrm{~b}$ & $113.68 \mathrm{~b}$ & $83.37 \mathrm{~b}$ & $93.45 \mathrm{~b}$ & $102.8 \mathrm{~b}$ & $115.37 \mathrm{~b}$ \\
\hline & $45 \mathrm{~N}$ & $93.87 \mathrm{a}$ & $117.30 \mathrm{a}$ & $135.14 \mathrm{a}$ & $159.14 \mathrm{a}$ & $109.33 \mathrm{a}$ & $135.65 \mathrm{a}$ & $147.13 \mathrm{a}$ & $180.41 \mathrm{a}$ \\
\hline
\end{tabular}

Means having the same letter within each column are not significantly differed at 0.05 level, according to Duncan's multiple range test. 
Table 8. Effect of interaction between genotypes and nitrogen levels on leaves fresh weight (g) of sunflower at 30, 40, 50 and 60 days after sowing in 2018 and 2019 seasons

\begin{tabular}{cccccccccc}
\hline Genotype Nitrogen level & \multicolumn{4}{c}{ Leaves fresh weight(g) 2018 } & \multicolumn{3}{c}{ Leaves fresh weight (g)2019 } \\
\hline \multicolumn{2}{c}{ Days } & $\mathbf{3 0}$ & $\mathbf{4 0}$ & $\mathbf{5 0}$ & $\mathbf{6 0}$ & $\mathbf{3 0}$ & $\mathbf{4 0}$ & $\mathbf{5 0}$ & $\mathbf{6 0}$ \\
\hline \multirow{2}{*}{ Sakha-53 } & $15 \mathrm{~N}$ & $80.47 \mathrm{e}$ & $84.18 \mathrm{f}$ & 98.51 & $114.32 \mathrm{~g}$ & $82.90 \mathrm{f}$ & $100.03 \mathrm{f}$ & $110.52 \mathrm{~g}$ & $124.4 \mathrm{~g}$ \\
& $30 \mathrm{~N}$ & $90.72 \mathrm{c}$ & $105.15 \mathrm{~d}$ & 116.1 & $133.5 \mathrm{f}$ & $97.29 \mathrm{~d}$ & $113.72 \mathrm{e}$ & $125.30 \mathrm{e}$ & $145.16 \mathrm{e}$ \\
& $45 \mathrm{~N}$ & $102.16 \mathrm{~b}$ & $125.43 \mathrm{~b}$ & 14530 & $190.07 \mathrm{~b}$ & $118.23 \mathrm{~b}$ & $155.16 \mathrm{~b}$ & $179.53 \mathrm{~b}$ & $218.99 \mathrm{~b}$ \\
& $15 \mathrm{~N}$ & $75.63 \mathrm{e}$ & $81.13 \mathrm{f}$ & 74.32 & $111.17 \mathrm{~g}$ & $77.53 \mathrm{f}$ & $96.83 \mathrm{f}$ & $106.33 \mathrm{~g}$ & $121.43 \mathrm{~g}$ \\
\multirow{2}{*}{ Line-120 } & $30 \mathrm{~N}$ & $84.72 \mathrm{c}$ & $100.11 \mathrm{~d}$ & 112.16 & $130.17 \mathrm{f}$ & $94.31 \mathrm{~d}$ & $107.22 \mathrm{e}$ & $120.17 \mathrm{e}$ & $140.13 . \mathrm{e}$ \\
& $45 \mathrm{~N}$ & $96.83 \mathrm{~b}$ & $125.43 \mathrm{~b}$ & 140.58 & $185.43 \mathrm{~b}$ & $113.26 \mathrm{~b}$ & $150.47 \mathrm{~b}$ & $175.73 \mathrm{~b}$ & $215.17 \mathrm{~b}$ \\
& $15 \mathrm{~N}$ & $82.71 \mathrm{~d}$ & $107.5 \mathrm{~d}$ & 129.16 & $153.9 \mathrm{~d}$ & $105.12 \mathrm{c}$ & $127.41 \mathrm{~d}$ & $145.18 \mathrm{~d}$ & $160.40 \mathrm{~d}$ \\
\multirow{2}{*}{ Giza-102 } & $30 \mathrm{~N}$ & $99.27 \mathrm{~b}$ & $122.11 \mathrm{c}$ & 150.40 & $175.98 \mathrm{c}$ & $115.23 \mathrm{~b}$ & $145.07 \mathrm{c}$ & $165.43 \mathrm{c}$ & $183.41 \mathrm{c}$ \\
& $45 \mathrm{~N}$ & $123.36 \mathrm{a}$ & $148.32 \mathrm{a}$ & $189.13 \mathrm{a}$ & $206.23 \mathrm{a}$ & $135.47 \mathrm{a}$ & $175.27 \mathrm{a}$ & $205.11 \mathrm{a}$ & $240.16 \mathrm{a}$
\end{tabular}

Means having the same letter within each column are not significantly differed at 0.05 level, according to Duncan's multiple range test.

Table 9. Effect of genotypes and nitrogen levels on leaves dry weight (g) of sunflower at 30, 40, 50 and 60 days after sowing in 2018 and 2019 seasons

\begin{tabular}{|c|c|c|c|c|c|c|c|c|c|}
\hline \multirow{2}{*}{$\begin{array}{l}\begin{array}{l}\text { Vegetative } \\
\text { characters }\end{array} \\
\\
\end{array}$} & \multirow{2}{*}{ Treatment } & \multicolumn{4}{|c|}{ Leaves dry weigh(g) 2018} & \multicolumn{4}{|c|}{ Leaves dry weight(g) 2019} \\
\hline & & 30 & 40 & 50 & 60 & 30 & 40 & 50 & 60 \\
\hline \multirow[t]{3}{*}{ Genotype } & Sakha-53 & $22.63 b$ & $28.16 \mathrm{~b}$ & $32.52 \mathrm{~b}$ & $34.09 \mathrm{~b}$ & $30.18 \mathrm{~b}$ & $31.17 \mathrm{~b}$ & $34.96 \mathrm{~b}$ & $43.15 \mathrm{~b}$ \\
\hline & Line-120 & $20.75 \mathrm{c}$ & $24.45 \mathrm{c}$ & $28.17 \mathrm{c}$ & $31.27 \mathrm{c}$ & $26.47 \mathrm{c}$ & $29.43 c$ & $33.84 \mathrm{c}$ & $39.34 \mathrm{c}$ \\
\hline & Giza-102 & $22.80 \mathrm{a}$ & $30.14 \mathrm{a}$ & $35.08 \mathrm{a}$ & $42.19 \mathrm{a}$ & $31.36 \mathrm{a}$ & $32.45 \mathrm{a}$ & $40.16 \mathrm{a}$ & $46.62 \mathrm{a}$ \\
\hline \multirow[t]{3}{*}{ Nitrogen level } & $15 \mathrm{~N}$ & $17.32 \mathrm{c}$ & $25.99 \mathrm{c}$ & $28.50 \mathrm{c}$ & $30.29 c$ & $27.92 \mathrm{c}$ & $29.71 \mathrm{c}$ & $31.05 \mathrm{c}$ & $34.06 \mathrm{c}$ \\
\hline & $30 \mathrm{~N}$ & $24.71 \mathrm{~b}$ & $26.59 \mathrm{~b}$ & $33.17 \mathrm{~b}$ & $36.20 \mathrm{~b}$ & $31.56 \mathrm{~b}$ & $32.18 \mathrm{~b}$ & $35.98 \mathrm{~b}$ & $43.63 \mathrm{~b}$ \\
\hline & $45 \mathrm{~N}$ & $19.63 \mathrm{a}$ & $33.41 \mathrm{a}$ & $38.04 \mathrm{a}$ & $44.84 \mathrm{a}$ & $34.16 \mathrm{a}$ & $35.96 \mathrm{a}$ & $46.11 \mathrm{a}$ & $55.46 \mathrm{a}$ \\
\hline
\end{tabular}

Means having the same letter within each column are not significantly differed at 0.05 level, according to Duncan's multiple range test.

cultivar gave the highest value of leaves dry weight $(22.80,30.14,35.08$ and $42.19 \mathrm{~g})$ in the first seasons, the same trend was recorded in the second season leaves dry weight valued as much as $(31.36,32.45$, 40.16 and $46.62 \mathrm{~g}$ ) at $30,40,50$ and 60 DAS, respectively.

\section{Effect of nitrogen levels}

There are significant effects of nitrogen levels on leaves dry weight $(\mathrm{g})$ at all growth stages studied of sunflower in both seasons (Table 9). The heights values of leaves dry weight $(19.63,33.41,38.04$ and 44.84) in the first seasons, as well as 34.16, 35.96, 46.11 and 55.46) in the second seasons at $30,40,50$ and $60 \mathrm{DAS}$, respectively.

\section{Effect of interaction}

Results in Table 10 show significant effect of genotypes $\mathrm{x}$ nitrogen levels interaction on leaves dry weight $(\mathrm{g})$ at 30 , 40, 50 and 60 DAS in 2018 and 2019 seasons. The largest leaves dry weight $(35.74,37.25,44.20$ and 55.87) in the first seasons, as well as in the second seasons $(39.74,42.54,56.18$ and 65.23) at 30, 40, 50 and 60 DAS, respectively were obtained 
Table10. Effect of interaction between genotypes and nitrogen levels on leaves dry weight (g) of sunflower at 30, 40, 50 and 60 days after sowing in 2018 and 2019 seasons

\begin{tabular}{|c|c|c|c|c|c|c|c|c|c|}
\hline \multirow{2}{*}{\multicolumn{2}{|c|}{$\begin{array}{c}\text { Genotype Nitrogen level } \\
\text { Days }\end{array}$}} & \multicolumn{4}{|c|}{ Leaves dry weight (g)2018 } & \multicolumn{4}{|c|}{ Leaves dry weight (g) 2019} \\
\hline & & 30 & 40 & 50 & 60 & 30 & 40 & 50 & 60 \\
\hline \multirow{4}{*}{ Sakha-53 } & $15 \mathrm{~N}$ & $26.14 \mathrm{e}$ & 30.93 ef & $35.91 \mathrm{f}$ & $35.28 \mathrm{gh}$ & 32.19 & $33.26 \mathrm{f}$ & $37.19 \mathrm{~g}$ & $50.61 \mathrm{c}$ \\
\hline & $30 \mathrm{~N}$ & $30.80 \mathrm{c}$ & $32.47 \mathrm{~d}$ & $36.15 \mathrm{e}$ & $42.64 \mathrm{~cd}$ & 33.81 & $35.15 \mathrm{e}$ & $39.65 \mathrm{f}$ & $54.45 \mathrm{~b}$ \\
\hline & $45 \mathrm{~N}$ & $32.73 \mathrm{~b}$ & $35.14 \mathrm{~b}$ & $42.12 \mathrm{~b}$ & $50.13 \mathrm{~b}$ & 37.60 & $40.29 \mathrm{~b}$ & $50.68 \mathrm{~b}$ & $65.20 \mathrm{a}$ \\
\hline & $15 \mathrm{~N}$ & $22.18 \mathrm{e}$ & $26.54 \mathrm{ef}$ & $32.41 \mathrm{e}$ & $38.29 \mathrm{~cd}$ & 29.89 & $30.93 \mathrm{f}$ & $33.15 \mathrm{~g}$ & $45.63 \mathrm{c}$ \\
\hline \multirow[t]{3}{*}{ Line-120 } & $30 \mathrm{~N}$ & $25.34 \mathrm{c}$ & $28.14 \mathrm{~d}$ & $37.53 b$ & $45.31 \mathrm{~b}$ & 30.74 & $31.18 \mathrm{e}$ & $36.46 \mathrm{f}$ & $50.19 \mathrm{~b}$ \\
\hline & $45 \mathrm{~N}$ & $27.13 b$ & $31.52 \mathrm{~b}$ & 24.131 & 21.091 & 32.24 & $35.62 b$ & $45.11 \mathrm{~b}$ & $60.71 \mathrm{a}$ \\
\hline & $15 \mathrm{~N}$ & $26.75 \mathrm{~d}$ & $31.12 \mathrm{e}$ & $37.05 \mathrm{~d}$ & $43.65 \mathrm{c}$ & 33.01 & $34.44 \mathrm{~d}$ & $45.17 \mathrm{~d}$ & $50.16 \mathrm{c}$ \\
\hline \multirow[t]{2}{*}{ Giza-102 } & $30 \mathrm{~N}$ & $30.19 \mathrm{c}$ & $33.45 \mathrm{c}$ & $38.79 \mathrm{c}$ & $47.93 \mathrm{~b}$ & 35.28 & $35.97 \mathrm{c}$ & $46.34 \mathrm{c}$ & $52.10 \mathrm{~b}$ \\
\hline & $45 \mathrm{~N}$ & $35.74 \mathrm{a}$ & $37.25 \mathrm{a}$ & $44.20 \mathrm{a}$ & $55.87 \mathrm{a}$ & $39.74 \mathrm{a}$ & $42.54 \mathrm{a}$ & $56.18 \mathrm{a}$ & $65.23 \mathrm{a}$ \\
\hline
\end{tabular}

Means having the same letter within each column are not significantly differed at 0.05 level, according to Duncan's multiple range test.

under the interaction effect of sunflower Giza-102- and $\mathrm{N}$ fertilizer level $45 \mathrm{~kg} / \mathrm{fad}$. The same results were found by Awad and Gharib (2009), Abd El-Motagally and Osman (2010), Hassan (2010), Ravishankar and Malligawad (2017) and Cechin et al. (2018). They reported that adding of sunflower seed production response to mineral nitrogen fertilizers level was followed by a significant increase in leaves dry weight.

\section{Yield and Yield Components}

Head diameter, 100 seed weight and seed oil content $(\%)$

\section{Genotypes variation}

There are significant differences among sunflower genotypes head diameter, 100 seed weight and seed oil content (\%) in both seasons (Table 11). Giza-102 gave the highest value for each of head diameter (cm) $(16.08,18.31), 100$-seed weight $(\mathrm{g})$ $(5.40,5.60)$ and seed oil content $(\%)(39.42$, 42.53) in the first and second seasons, respectively.

\section{Effect of nitrogen levels}

There are significant effects of nitrogen levels on head diameter, 100 seed weight and seed oil content $(\%)$ in both seasons (Table
11). The heights values of head diameter (cm) $(17.90,18.23), 100$-seed weight $(\mathrm{g})$ $(5.71,6.72)$ and seed oil content (\%) $(34.45,34.33)$ in the first and second season, respectively were obtained due to applying $45 \mathrm{kgN} / \mathrm{fad}$.

\section{Effect of interaction}

Results in Table 12 show significant effect of genotypes $x$ nitrogen levels interaction on head diameter, 100 seed weight and seed oil content (\%) in 2018 and 2019 seasons. There were significant effects of sunflower Genotype $\mathrm{x}$ nitrogen levels interaction. The tallest head diameter $(\mathrm{cm})$ (21.07, 22.56), 100-seed weight (g) (7.49, $8.02)$ and seed oil content $(\%)(32.25,33.63)$ in the first and second season, respectively were obtained under the interaction effect of sunflower Giza-102- and $\mathrm{N}$ fertilizer level $45 \mathrm{~kg} / \mathrm{fad}$. Similar studied have been reported by El-Sarag (2007), Awad and Ghrib (2009), Solimanzadeh et al. (2010), Mahrous et al. (2014), Gul and Kara (2015) and Kandil et al. (2017). Seed weight (g/plant) and seed and oil yields/fad.

\section{Genotypes variation}

There are significant differences among sunflower genotypes head diameter, 100 seed weight and seed oil content (\%) in both 
Table 11. Effect of genotypes and nitrogen levels on head diameter (cm), 100-seed weight (gm) and seed oil content $(\%)$ of sunflower in 2018 and 2019 seasons

\begin{tabular}{|c|c|c|c|c|c|c|c|}
\hline \multirow{2}{*}{$\begin{array}{l}\text { Vegetative } \\
\text { characters }\end{array}$} & \multirow[t]{2}{*}{ Treatment } & \multicolumn{2}{|c|}{ Head diameter $(\mathrm{cm})$} & \multicolumn{2}{|c|}{ 100-seed weight (g) } & \multicolumn{2}{|c|}{ Seed oil content (\%) } \\
\hline & & 2018 & 2019 & 2018 & 2019 & 2018 & 2019 \\
\hline \multirow[t]{3}{*}{ Genotype } & Sakha-53 & $16.00 \mathrm{ab}$ & $17.92 \mathrm{ab}$ & $3.71 \mathrm{~b}$ & $5.28 \mathrm{~b}$ & $37.46 \mathrm{~b}$ & $35.59 \mathrm{~b}$ \\
\hline & Line-120 & $15.99 \mathrm{~b}$ & $18.15 b$ & $3.26 \mathrm{c}$ & $5.10 \mathrm{c}$ & $35.63 \mathrm{c}$ & $37.74 \mathrm{c}$ \\
\hline & Giza-102 & $16.08 \mathrm{a}$ & $18.31 \mathrm{a}$ & $5.40 \mathrm{a}$ & $5.60 \mathrm{a}$ & $39.42 \mathrm{a}$ & $42.53 \mathrm{a}$ \\
\hline \multirow[t]{3}{*}{ Nitrogen level } & $15 \mathrm{~N}$ & $14.20 \mathrm{~b}$ & $18.08 \mathrm{~b}$ & $3.45 \mathrm{c}$ & $4.21 \mathrm{c}$ & $42.49 \mathrm{a}$ & $43.72 \mathrm{a}$ \\
\hline & $30 \mathrm{~N}$ & $15.98 \mathrm{ab}$ & $18.07 \mathrm{~b}$ & $4.50 \mathrm{~b}$ & $5.41 \mathrm{~b}$ & $38.38 \mathrm{~b}$ & $39.12 \mathrm{~b}$ \\
\hline & $45 \mathrm{~N}$ & $17.90 \mathrm{a}$ & $18.23 \mathrm{a}$ & $5.71 \mathrm{a}$ & $6.72 \mathrm{a}$ & $34.45 \mathrm{c}$ & $34.33 \mathrm{c}$ \\
\hline
\end{tabular}

Means having the same letter within each column are not significantly differed at 0.05 level, according to Duncan's multiple range test.

Table 12. Effect of interaction between genotypes and nitrogen levels on head diameter (cm), 100-seed weight (g) and seed oil content (\%) of sunflower in 2018 and 2019 seasons

\begin{tabular}{lcllllll}
\hline $\begin{array}{l}\text { Genotype } \\
\text { Seasons }\end{array}$ & Nitrogen level & \multicolumn{2}{c}{ Head diameter $(\mathbf{c m})$} & \multicolumn{2}{c}{ 100-seed weight $(\mathbf{g})$} & \multicolumn{2}{c}{ Seed oil content (\%) } \\
\cline { 3 - 8 } Sakha-53 & & $\mathbf{2 0 1 8}$ & $\mathbf{2 0 1 9}$ & $\mathbf{2 0 1 8}$ & $\mathbf{2 0 1 9}$ & $\mathbf{2 0 1 8}$ & $\mathbf{2 0 1 9}$ \\
\hline & $15 \mathrm{~N}$ & $16.13 \mathrm{c}-\mathrm{f}$ & $16.87 \mathrm{ghi}$ & $4.24 \mathrm{j}$ & $5.93 \mathrm{e}$ & $38.18 \mathrm{ab}$ & $39.87 \mathrm{bc}$ \\
& $30 \mathrm{~N}$ & $18.00 \mathrm{~b}$ & $18.40 \mathrm{~b}-\mathrm{f}$ & $4.54 \mathrm{~h}$ & $6.31 \mathrm{~d}$ & $38.18 \mathrm{ab}$ & $39.87 \mathrm{bc}$ \\
& $45 \mathrm{~N}$ & $20.23 \mathrm{a}$ & $19.53 \mathrm{ab}$ & $5.45 \mathrm{e}$ & $7.13 \mathrm{~b}$ & $38.18 \mathrm{ab}$ & $39.87 \mathrm{bc}$ \\
Line-120 & $15 \mathrm{~N}$ & $16.20 \mathrm{c}-\mathrm{f}$ & $17.33 \mathrm{f}-\mathrm{i}$ & $3.97 \mathrm{j}$ & $5.71 \mathrm{e}$ & $34.27 \mathrm{~cd}$ & $36.01 \mathrm{ef}$ \\
& $30 \mathrm{~N}$ & $17.60 \mathrm{bc}$ & $18.00 \mathrm{c}-\mathrm{h}$ & $4.35 \mathrm{~h}$ & $6.18 \mathrm{~d}$ & $34.27 \mathrm{~cd}$ & $36.01 \mathrm{ef}$ \\
& $45 \mathrm{~N}$ & $18.23 \mathrm{~b}$ & $19.00 \mathrm{abc}$ & $5.27 \mathrm{e}$ & $7.09 \mathrm{~b}$ & $34.27 \mathrm{~cd}$ & $36.01 \mathrm{ef}$ \\
Giza-102 & $15 \mathrm{~N}$ & $16.40 \mathrm{c}-\mathrm{f}$ & $16.80 \mathrm{hi}$ & $6.14 \mathrm{c}$ & $6.24 \mathrm{~d}$ & $32.25 \mathrm{e}$ & $33.63 \mathrm{~g}$ \\
& $30 \mathrm{~N}$ & $17.23 \mathrm{bcd}$ & $18.67 \mathrm{a}-\mathrm{e}$ & $6.52 \mathrm{~b}$ & $6.62 \mathrm{c}$ & $32.25 \mathrm{e}$ & $33.63 \mathrm{~g}$ \\
& $45 \mathrm{~N}$ & $21.07 \mathrm{a}$ & $22.56 \mathrm{a}$ & $7.49 \mathrm{a}$ & $8.02 \mathrm{a}$ & $32.25 \mathrm{e}$ & $33.63 \mathrm{~g}$
\end{tabular}

Means having the same letter within each column are not significantly differed at 0.05 level, according to Duncan's multiple range test.

seasons (Table 13). Giza-102 gave the highest value for each of seed weight/ plant (26.42, $44.75 \mathrm{~g})$, seed yield $(1.056,1.790$ $\mathrm{kg} / \mathrm{fad}$.), and oil yield (241.4, $\left.394.3 \mathrm{~kg} \mathrm{fad}^{-1}\right)$ in the first and second seasons, respectively.

\section{Effect of nitrogen levels}

There are significant effects of nitrogen levels on head diameter, 100 seed weight and seed oil content (\%) in both seasons (Table 13). The heights values of seed weight/plant $(25.18,48.75 \mathrm{~g})$, seed yield (1.007, $1.950 \mathrm{~kg} / \mathrm{fad}$.$) , and oil yield (288.1,$ $\left.477.2 \mathrm{~kg} \mathrm{fad} .^{-1}\right)$ in the first and second season, respectively were obtained when $45 \mathrm{~kg} \mathrm{~N} / \mathrm{fad}$.

\section{Effect of interaction}

Results in Table 14 show significant effect of genotypes $\mathrm{x}$ nitrogen levels interaction on head diameter, 100 seed weight and seed oil content (\%) in 2018 and 2019 seasons. The highest on seed weight/ plant (26.97, 42.68 g), seed yield (1.078, $1.707 \mathrm{~kg} / \mathrm{fad}$.$) , and oil$ yield $\left(0.797,0.830 \mathrm{~kg} \mathrm{fad}^{-1}\right)$ in the first and second season, were recorded due to the interaction effect of Giza-102 cultivar and $\mathrm{N}$ fertilizer level $45 \mathrm{~kg} / \mathrm{fad}$. The same results were found by El-Aref et al. (2011), Maqsood (2015) Shehzad and Maqsood (2015), Dhillon et al. (2017), Bagheri et al. (2018) and Schultz et al. (2018). 
Table 13. Effect of genotypes and nitrogen levels on seed weight/plant (g), seed yield (kg/ fad.), and oil yield (kg fad. ${ }^{-1}$ ) of sunflower in 2018 and 2019 seasons

\begin{tabular}{|c|c|c|c|c|c|c|c|}
\hline \multicolumn{2}{|c|}{ Vegetative characters } & \multirow{2}{*}{\multicolumn{4}{|c|}{ Seed weight/ plant(g) Seed yield (kg/fad.) }} & \multicolumn{2}{|c|}{ Oil yield $\left(\mathrm{kg} \mathrm{fad}^{-1}\right)$} \\
\hline & eatment & & & & & & \\
\hline & & 2018 & 2019 & 2018 & 2019 & 2018 & 2019 \\
\hline \multirow[t]{3}{*}{ Genotype } & Sakha-53 & $23.27 \mathrm{ab}$ & $39.42 \mathrm{ab}$ & $0.930 \mathrm{ab}$ & $1.570 \mathrm{ab}$ & $158.8 \mathrm{~b}$ & $238.3 \mathrm{~b}$ \\
\hline & Line-120 & $21.97 \mathrm{~b}$ & $35.86 \mathrm{~b}$ & $0.878 \mathrm{c}$ & $1.430 \mathrm{c}$ & $152.80 \mathrm{c}$ & $230.88 \mathrm{c}$ \\
\hline & Giza-102 & $26.42 \mathrm{a}$ & $44.75 \mathrm{a}$ & $1.056 \mathrm{a}$ & $1.790 \mathrm{a}$ & $241.4 \mathrm{a}$ & $394.3 \mathrm{a}$ \\
\hline \multirow[t]{3}{*}{ Nitrogen level } & $15 N$ & $22.07 \mathrm{c}$ & $38.96 \mathrm{c}$ & $0.882 \mathrm{c}$ & $1.550 \mathrm{c}$ & $123.7 \mathrm{c}$ & $179.3 \mathrm{c}$ \\
\hline & $30 \mathrm{~N}$ & $24.50 \mathrm{~b}$ & $41.18 \mathrm{~b}$ & $0.980 \mathrm{~b}$ & $1.640 \mathrm{~b}$ & $188.7 \mathrm{~b}$ & $292.4 \mathrm{~b}$ \\
\hline & $45 \mathrm{~N}$ & $25.18 \mathrm{a}$ & $48.75 \mathrm{a}$ & $1.007 \mathrm{a}$ & $1.950 \mathrm{a}$ & $288.1 \mathrm{a}$ & $477.2 \mathrm{a}$ \\
\hline
\end{tabular}

Means having the same letter within each column are not significantly differed at 0.05 level, according to Duncan's multiple range test.

Table14. Effect of interaction between genotypes and nitrogen levels on seed weight/ plant $(\mathrm{g})$, seed yield (kg/fad.), and oil yield (kg fad..$^{-1}$ ) of sunflower in 2018 and 2019 seasons

\begin{tabular}{lccccccc}
\hline Genotype Nitrogen level & \multicolumn{2}{c}{ Seed weight/plant(g) } & \multicolumn{2}{c}{ Seed yield (kg/fad.) } & \multicolumn{2}{c}{ Oil yield (kg fad. ${ }^{-1}$ ) } \\
\hline \multirow{2}{*}{ Sakha-53 } & & $\mathbf{2 0 1 8}$ & $\mathbf{2 0 1 9}$ & $\mathbf{2 0 1 8}$ & $\mathbf{2 0 1 9}$ & $\mathbf{2 0 1 8}$ & $\mathbf{2 0 1 9}$ \\
& $15 \mathrm{~N}$ & $20.52 \mathrm{~g}$ & $35.44 \mathrm{~h}$ & $0.820 \mathrm{~g}$ & $1.417 \mathrm{~h}$ & $0.736 \mathrm{abc}$ & $0.769 \mathrm{abc}$ \\
& $30 \mathrm{~N}$ & $22.28 \mathrm{f}$ & $37.11 \mathrm{f}$ & $0.891 \mathrm{f}$ & $1.484 \mathrm{f}$ & $0.837 \mathrm{ab}$ & $0.876 \mathrm{ab}$ \\
& $45 \mathrm{~N}$ & $25.18 \mathrm{~b}$ & $41.36 \mathrm{~d}$ & $1.007 \mathrm{~b}$ & $1.654 \mathrm{~d}$ & $0.787 \mathrm{ab}$ & $0.861 \mathrm{ab}$ \\
\multirow{2}{*}{ Line-120 } & $15 \mathrm{~N}$ & $19.96 \mathrm{~g}$ & 34.21 & 0.798 & 1.368 & $0.627 \mathrm{bcd}$ & $0.660 \mathrm{~b}-\mathrm{e}$ \\
& $30 \mathrm{~N}$ & $21.84 \mathrm{f}$ & 36.18 & 0.873 & 1.447 & $0.588 \mathrm{~b}-\mathrm{h}$ & $0.589 \mathrm{~b}-\mathrm{h}$ \\
& $45 \mathrm{~N}$ & $24.61 \mathrm{~b}$ & 40.61 & 0.984 & 1.624 & $0.567 \mathrm{~b}-\mathrm{g}$ & $0.597 \mathrm{~b}-\mathrm{g}$ \\
\multirow{2}{*}{ Giza-102 } & $15 \mathrm{~N}$ & $21.16 \mathrm{c}$ & $36.92 \mathrm{c}$ & $0.846 \mathrm{c}$ & $1.476 \mathrm{c}$ & $0.554 \mathrm{~b}-\mathrm{f}$ & $0.579 \mathrm{~b}-\mathrm{f}$ \\
& $30 \mathrm{~N}$ & $23.76 \mathrm{~b}$ & $38.84 \mathrm{~b}$ & $0.950 \mathrm{~b}$ & $1.553 \mathrm{~b}$ & $0.580 \mathrm{~b}-\mathrm{e}$ & $0.605 \mathrm{bcd}$ \\
& $45 \mathrm{~N}$ & $26.97 \mathrm{a}$ & $42.68 \mathrm{a}$ & $1.078 \mathrm{a}$ & $1.707 \mathrm{a}$ & $0.797 \mathrm{a}$ & $0.830 \mathrm{a}$ \\
\hline
\end{tabular}

Means having the same letter within each column are not significantly differed at 0.05 level, according to Duncan's multiple range test.

\section{REFERENCES}

Abaza, G.M.S.M. (2010). Effect of some agricultural practices on some sunflower genotype characters induced by gamma irrigation, M.Sc. Thesis, Crop Sci. Dept., Fac. Agric., Zagazig Univ.

Abd El-Motagally, F.M.F. and Osman, E.A. (2010). Effect of nitrogen and potassium fertilization combinations on productivity of two Sunflower cultivars under East of El-Ewinate conditions.
Ame.-Euras. J. Agric. Environ. Sci., 8 (4): 397-401.

Awad, M.M. and Gharib, H.S. (2009). Productivity of some open pollinated sunflower populations under different nitrogen fertilizer rates in North Delta. J. Agric. Res., 35(2): 503-521.

AOAC (1990). Official Method of analysis, kjeldahl method No. 984.13, Chapter 4, p. $31,19^{\text {th }}$ Ed. Tecator Application Notes AN 300. 
Bagheri, F.; Kazemeini, S.A.; Bahrani, M.J. and Heidari, B. (2018). Effect of nitrogen, wheat residues, and compost rates on the growth and yield of sunflower. Ukrainian J. Ecol., 8 (1):736744.

Braga, D.F.; Oliveira, F.H.T.; Santos, H.C.; Araújo, A.P. and Zonta, E. (2018). Nitrogen and phosphorus fertilization of sunflower crop in alkaline Cambisol. Revista Brasileira de Engenharia Agricola e Ambiental, 22 (2): 101-106.

Cechin, I.; Gonzalez, G.C.; Corniani, N. and Fumis, T.D.F. (2018). The sensitivity of sunflower (Helianthus annuus L.) plants to UV-B radiation is altered by nitrogen status. Santa Maria, 48(2):1-6.

Dhillon, B.S.; Sharma, P.; Sharma, S. and Sharma, S. (2017). Oil yield and fatty acid composition of spring sunflower as affected by sowing date, Intra row spacing and nitrogen dose. Indian J. Agric. Biochem., 30 (2): 135-140.

Duncan, D.B. (1955). Multiple Range and Multiple F. test. Biometrics, (11):1-24.

El-Aref, Kh.A.O.; Abo-El-Hamd, A.S.A. and Abd El-Monem, A.M.A. (2011). Influence of filter MUC cake fertilization under low levels of nitrogen on yield and its components for tow sunflower cultivars. Mansoura J. Plant Prod., 2 (2):165 - 178.

El-Sarag, E.I. (2007). Influence of plant population and nitrogen fertilization levels on performance of some sunflower cultivars under North Sinai condition. Ann. Agric. Sci., Ain Shams Univ., Cairo, 25(1):113-121.

Gul, V. and Kara, K. (2015). Effects of different nitrogen doses on yield and quality traits of common sunflower (Helianthus annuus L.). Turkish J. Field Crops, 20 (2):159-165.

Hama, S.J. (2015). Response of growth, yield and yield components of two sunflower varieties (Helianthus annuиs L.) to nitrogen fertilization. J. Zankoi Sulaimani, 17(4):239-250.

Hassan, T.H.A. (2010). Bio and mineral fertilization studies on sunflower under North Sinai conditions. Ph.D. Thesis, Fac. Environ. Agric. Sci., Suez Canal Univ.

Irika, M.A. (2015). Effect of organic and inorganic nitrogen sources on growth, yield and oil content of Sunflower grown in highly weathered soils of Morogoro. M.Sc. Thesis, Fac. Agric., Nairobi Univ.

Kandil, A.A.; Sharief, A.E. and Odam, A.M.A. (2017). Response of some sunflower hybrids (Helianthus annuus L.) to different nitrogen fertilizer rates and plant densities. Int. J. Environ., Agric. and Biotechnol., 2(6):1870-1878.

Nasim, W.; Ahmad, A.; Hammad, H.M.; Chaudhary, H.J. and Munis, M.F.H. (2012). Effect of nitrogen on growth and yield of sunflower under semi-arid conditions of Pak. Bot., 44 (2): 639- 648.

Oyinlola, E.Y.; Ogunwole, J.O. and Amapu, I.Y. (2010). Response of sunflower (Helianthus annuus L.) to nitrogen application in A Savanna Al Fisol. Helia, 33 (52):115-126.

Ravishankar, G. and Malligawad, L.H. (2017). Response of sunflower to different N/P fertilizer ratios and levels of nitrogen and phosphorus. Int. J. Current Microbiol. and Appl. Sci., 6 (8): 980-986.

Schultz, E.; DeSutter, T.; Sharma, L.; Endres, G.; Ashley, R.; Bu, H.; Markell, S.; Kraklau, A. and Franzen, D. (2018). Response of sunflower to nitrogen and phosphorus in North Dakota. Agron. J., 110 (2): 1-11.

Snedecor, G.W. and Cochran, W.G. (1956). Statistical Methods Applied to Experiments in Agriculture and Biology. $5^{\text {th }}$ Ed. Ames, Iowa: Iowa State Univ. 


$$
\begin{aligned}
& \text { الملخص العربي } \\
& \text { إستجابة بعض التراكيب الوراثية لدوار الثمس لمستويات السماد النيتروجيني }
\end{aligned}
$$

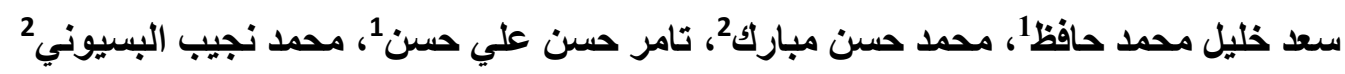

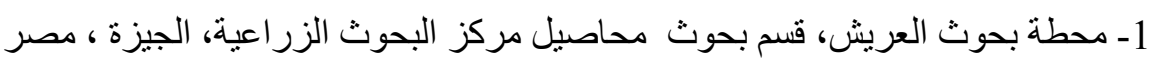

$$
\begin{aligned}
& \text { 2- قسم الإنتاج النباتي، كلية العلوم الزر اعية البيئية ، جامعة العريش، مصر }
\end{aligned}
$$

أجريت دراسة حقلية خلال الموسم الصيفي لعامي 2018 و 2019م في المزر عة التجريبية بكلية العلوم الزراعية البيئية

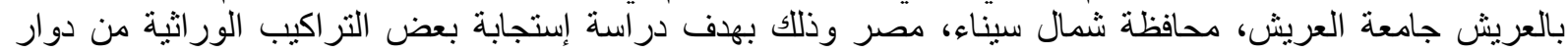
الثمس (سخا 53 وجيزة 102 وسلالة الثريت 120) لثلاث معدلات من التسميد النيتروجيني (45,30,15 كجم نيتروجين للفدان)

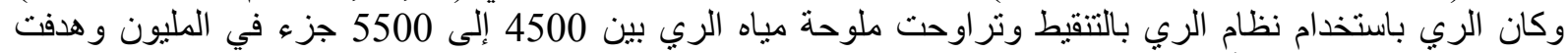

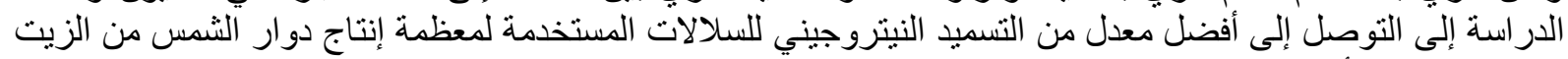

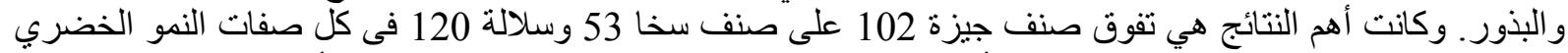
(ارتفاع النبات/سم، قطر الساق/سم، عدد أوراق النبات/نبات، الوزن الخضري الفرة والجاف للأوراق/جم)، ومساهمات

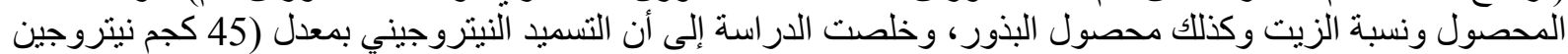

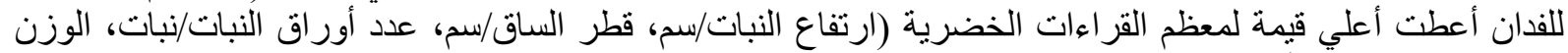

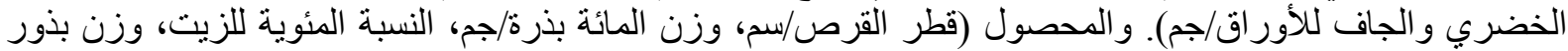

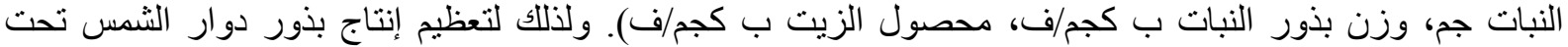
ظروف المناطق شبة الجافة يمكن زر اعة الصنف جيزة 102 مع معاملته بالتسميد النتروجيني 45 كجم نيتروجين للفدان. الكثمات الإسترشادية: التر اكيب الور اثية، دوار الثمس، التسميد النيتروجيني.

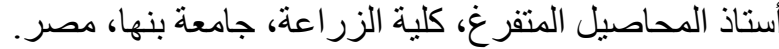
أستاذ المحاصيل، كلية العلوم الزراعية البيئية، جامعة العريش، الزراءة مصر.

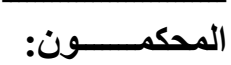

